\title{
Extending the radio spectrum of magnetic chemically peculiar stars to the $\mathrm{mm}$ range ${ }^{\star}$
}

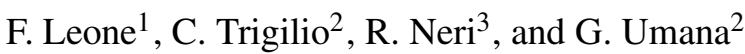 \\ 1 INAF - Osservatorio Astrofisico di Catania, Via S. Sofia n. 78, 95123 Catania, Italy \\ e-mail: fleone@ct.astro.it \\ 2 Istituto di Radioastronomia del C.N.R., sezione di Noto, PO Box 141, 96017 Noto, Italy \\ e-mail: [c.trigilio;g.umana]@ira.cnr.it \\ 3 Institut de Radioastronomie Millimètrique, 300 rue de la Piscine, 38406 Saint Martin d'Hères, France \\ e-mail: neri@iram.fr
}

Received 24 October 2003 / Accepted 14 May 2004

\begin{abstract}
Magnetic chemically peculiar (MCP) stars can present radio emission at centimetre wavelengths. The steep decrement of the dominant dipolar component of the photospheric magnetic field results in each radio frequency being mainly emitted in a well localised shell of the circumstellar region. To explore the most internal regions of the magnetosphere, observations of a sample of eleven MCP stars known to be radio sources in the $1.4-22.5 \mathrm{GHz}$ range were carried out at $87.7 \mathrm{GHz}$ with the IRAM interferometer. Millimeter emission, with a flux density at about $4 \times$ the sensitivity limit of our observations, was detected towards two of the stars: HD 35298 and HD 124224.

Combining our mm-observations with previous cm-observations, it appears that MCP stars with a relatively weak magnetic field present a radio spectrum that increases with frequency up to $22.5 \mathrm{GHz}$ and then decreases towards the mm range. In presence of strong fields, the radio spectrum is always decreasing with frequency.

A comparison of the observed cm-mm spectrum of HD 124224 with results of numerical simulations of the gyrosynchrotron emission suggests that circumstellar regions emitting in the mm-range cannot present magnetic fields larger than 1-2 kG.
\end{abstract}

Key words. stars: chemically peculiar - stars: individual: HD 35298, HD 124224 - stars: circumstellar matter stars: magnetic field - radio continuum: stars

\section{Introduction}

Magnetic Chemically Peculiar (MCP) stars can be radio sources in the centimetre range (Cassinelli 1984). Based on the low expected mass-loss rates, Drake et al. (1987) ruled out freefree emission from the stellar wind and related the radio emission to the presence of magnetic fields. Linsky et al. (1992) suggested that the radio emission is due to optically-thick gyrosynchrotron emission from non-thermal electrons. The photospheric magnetic field of MCP stars is mainly dipolar with a polar strength of a few $\mathrm{kG}$. As a result of the steep drop of the magnetic field strength with distance from the star, each observed radio frequency is mainly emitted in a well localised circumstellar shell, with the highest frequencies coming from the inner magnetosphere.

With the aim of studying the properties of the radio emitting regions, particularly the innermost regions, we have carried out observations at $87.7 \mathrm{GHz}$ with the IRAM interferometer of a sample of MCP stars. This attempt follows

* Based on observations carried out with the IRAM Plateau de Bure Interferometer. IRAM is supported by INSU/CNRS (France), MPG (Germany) and IGN (Spain).
SEST observations at $230 \mathrm{GHz}$ carried out in 1993 (Leone et al. 1996) on HD 124224, HD 142301 and HD 171247 when only upper limits, 2-3 times larger than the $5 \mathrm{GHz}$ flux, could be determined.

\section{IRAM observations and data reduction}

Eleven MCP stars known to be radio sources in the centimetre range (Table 1) were observed with the IRAM interferometer (Guilloteau et al. 1992) from May to December 1997 at $87.7 \mathrm{GHz}$. Visibilities were obtained for the most part with four antennas in the $\mathrm{CD}$ set of configurations. The crosscorrelator was adjusted to a contiguous bandwidth of $450 \mathrm{MHz}$. All the observations were performed using on-source integration times of $20 \mathrm{~min}$ interlaced with 4 min integrations of nearby quasars in order to calibrate instrumental amplitude and phase. For each star, the distance between the adopted coordinates (Table 1) for pointing and the values listed in the Hipparcos or Tycho catalogue (ESA 1997), after the correction for proper motions, is smaller than $20 \%$ of the beam-size.

Data calibration was carried out in the standard antennabased manner. Only visibilities with error $\leq 40$ degrees were 
Table 1. List of the magnetic chemically peculiar stars (HD number) observed with the IRAM interferometer at $87.7 \mathrm{GHz}$. We report the coordinates adopted for pointing, the measured flux densities at the Hipparcos or Tycho position and the associated error ( $\mathrm{rms}$ of the real part of visibilities). If available, the polar value $\left(B_{\mathrm{p}}\right)$ of the magnetic dipole is also quoted. Only HD 35298 and HD 124224 present flux densities in the mm-range about at four times the noise level.

\begin{tabular}{c|cc|cr|c}
\hline \hline \multicolumn{1}{c|}{$\begin{array}{c}\text { Star } \\
\text { HD }\end{array}$} & \multicolumn{2}{|c|}{$\begin{array}{c}\text { (2000) } \\
\text { hh }: \mathrm{mm}: \mathrm{ss}\end{array}$} & \multicolumn{1}{c|}{$\begin{array}{c}\text { hh }: \mathrm{mm}: \mathrm{ss} \\
\text { [mJy] }\end{array}$} & $\begin{array}{c}\mathrm{rms} \\
{[\mathrm{mJy}]}\end{array}$ & $\begin{array}{c}B_{\mathrm{p}} \\
\mathrm{kG}\end{array}$ \\
\hline 35298 & $05: 23: 50.367$ & $+02: 04: 55.70$ & $\mathbf{1 . 6 1}$ & 0.43 & \\
36485 & $05: 32: 00.380$ & $-00: 17: 04.30$ & 0.79 & 0.41 & \\
37017 & $05: 35: 21.872$ & $-04: 29: 39.09$ & 0.28 & 0.39 & $6.8^{a}$ \\
37479 & $05: 38: 47.060$ & $-02: 35: 38.90$ & 1.03 & 0.38 & $7.7^{a}$ \\
37808 & $05: 40: 46.134$ & $-10: 24: 32.66$ & 0.36 & 0.29 & \\
124224 & $14: 12: 15.807$ & $+02: 24: 33.91$ & $\mathbf{1 . 3 2}$ & 0.31 & $4.5^{b}$ \\
142301 & $15: 54: 39.557$ & $-25: 14: 37.36$ & 1.28 & 0.67 & $14.7^{c}$ \\
142990 & $15: 58: 34.80$ & $-24: 49: 51.7$ & -0.54 & 0.71 & $5.0^{d}$ \\
144334 & $16: 06: 06.396$ & $-23: 36: 23.04$ & 0.92 & 0.97 & \\
171247 & $18: 33: 23.270$ & $+08: 16: 05.40$ & 0.03 & 0.29 & \\
215441 & $22: 44: 07.370$ & $+55: 35: 21.20$ & 0.34 & 0.17 & $62.4^{e}$ \\
\hline
\end{tabular}

${ }^{a}$ Leone (1991).

${ }^{b}$ Trigilio et al. (2000).

${ }^{c}$ Landstreet et al. (1979).

${ }^{d}$ Bohlender et al. (1993).

${ }^{e}$ Landstreet \& Mathys (2000).

retained to estimate the flux density in the millimetre continuum. The absolute flux density scale was based on the radiostar MWC $349(0.95 \mathrm{Jy}$ at $87.7 \mathrm{GHz})$. When MWC 349 was not available, the scale was bootstrapped using a set of strong quasars whose flux densities are monitored regularly at Plateau de Bure. Globally, the flux density scale is estimated to be accurate to about $10 \%$ for each epoch. The receiver passband shape was determined on 3C273 and was estimated with sufficient accuracy $(\leq 1 \%)$.

Table 2 provides the details of the chronological logbook of the interferometric observations: number of antennas, minimum and maximum baseline and the Equivalent On-source Integration Time (EOIT) for an equivalent two-antenna array.

Of the observed stars, only HD 35298 and HD 124224 exhibit flux densities at about four times the noise level: $1.61 \pm$ 0.43 and $1.32 \pm 0.31 \mathrm{mJy}$, respectively. Errors are estimated as the rms of the real part of visibilities. For these two stars, Fig. 1 shows the obtained maps with the synthesized beams.

As to the undetected stars, Table 1 reports the measured fluxes at the Hipparcos or Tycho positions and the rms of the real part of visibilities.

In the gyrosynchrotron radiation model, frequency is proportional to the magnetic field strength, so that the highest observable frequency depends on the field strength at the stellar surface (Table 1). We focused our attention on HD 215441, the MCP star with the strongest known magnetic field. Landstreet \& Mathys (2000) modelled its field variation with the
Table 2. Log of IRAM 1997 observations. For each date, we report the number of antenna used $(N)$, the minimum and maximum baseline (rounded to $5 \mathrm{~m}$ ), the observed stars (HD number) and the equivalent on-source integration time (EOIT) for an equivalent two-antenna array.

\begin{tabular}{|c|c|c|c|c|c|}
\hline \multicolumn{2}{|c|}{ Date } & \multirow{2}{*}{$\begin{array}{l}N \\
4\end{array}$} & \multirow{2}{*}{$\begin{array}{c}\begin{array}{c}\text { Min \& max } \\
\text { baseline (m) }\end{array} \\
15-140\end{array}$} & \multirow{2}{*}{$\begin{array}{c}\text { Star } \\
\text { HD }\end{array}$} & \multirow{2}{*}{$\begin{array}{c}\begin{array}{c}\text { EOIT } \\
(\mathrm{h})\end{array} \\
14.0\end{array}$} \\
\hline May & 15 & & & & \\
\hline & 16 & 4 & $15-175$ & 171247 & 31.5 \\
\hline & & & & 215441 & 10.7 \\
\hline & 17 & 5 & $20-175$ & 215441 & 20.0 \\
\hline & 21 & 5 & $25-175$ & 142301 & 11.3 \\
\hline \multirow[t]{8}{*}{ June } & 7 & 5 & $20-225$ & 215441 & 9.9 \\
\hline & 8 & 5 & $20-270$ & 171247 & 8.7 \\
\hline & & & & 215441 & 16.6 \\
\hline & 10 & 4 & $20-165$ & 215441 & 3.3 \\
\hline & 13 & 3 & $30-65$ & 171247 & 8.0 \\
\hline & & & & 215441 & 12.0 \\
\hline & 25 & 3 & $20-45$ & 215441 & 2.0 \\
\hline & 28 & 3 & $15-45$ & 215441 & 6.0 \\
\hline \multirow[t]{9}{*}{ July } & 1 & 4 & $15-50$ & 215441 & 16.0 \\
\hline & 6 & 4 & $15-65$ & 124224 & 14.7 \\
\hline & & & & 142301 & 7.0 \\
\hline & & & & 144334 & 4.3 \\
\hline & & & & 142990 & 8.0 \\
\hline & 19 & 4 & $50-175$ & 124224 & 10.7 \\
\hline & 21 & 4 & $30-170$ & 144334 & 2.0 \\
\hline & 24 & 4 & $30-175$ & 142990 & 8.0 \\
\hline & & & & 215441 & 21.3 \\
\hline \multirow[t]{3}{*}{ Aug. } & 13 & 4 & $40-175$ & 171247 & 11.3 \\
\hline & 14 & 4 & $40-175$ & 215441 & 16.0 \\
\hline & 21 & 3 & $105-140$ & 36485 & 4.0 \\
\hline Sep. & 18 & 4 & $15-45$ & 124224 & 4.2 \\
\hline \multirow[t]{15}{*}{ Oct. } & 2 & 4 & $15-65$ & 35298 & 2.4 \\
\hline & & & & 36485 & 8.0 \\
\hline & & & & 37017 & 8.0 \\
\hline & & & & 37479 & 8.0 \\
\hline & & & & 37808 & 8.0 \\
\hline & 4 & 4 & $15-60$ & 35298 & 5.4 \\
\hline & & & & 37808 & 8.0 \\
\hline & 10 & 4 & $15-290$ & 35298 & 2.0 \\
\hline & & & & 36485 & 3.3 \\
\hline & & & & 37017 & 0.7 \\
\hline & & & & 37479 & 1.3 \\
\hline & & & & 37808 & 2.0 \\
\hline & 22 & 4 & $30-180$ & 37017 & 4.0 \\
\hline & & & & 37479 & 2.0 \\
\hline & & & & 37808 & 2.4 \\
\hline Nov. & 30 & 5 & $20-65$ & 35298 & 13.3 \\
\hline \multirow[t]{5}{*}{ Dec. } & 8 & 4 & $15-70$ & 36485 & 8.0 \\
\hline & & & & 37017 & 8.0 \\
\hline & & & & 37479 & 8.0 \\
\hline & & & & 37808 & 8.0 \\
\hline & 10 & 4 & $20-60$ & 215441 & 8.0 \\
\hline
\end{tabular}

rotational period under the assumption of an axisymmetric multipole expansion up to the octupole term and with dipole, quadrupole and octupole strengths equal to $+62.4,-42.0$ and $+24.2 \mathrm{kG}$, respectively. Despite its very strong magnetic 

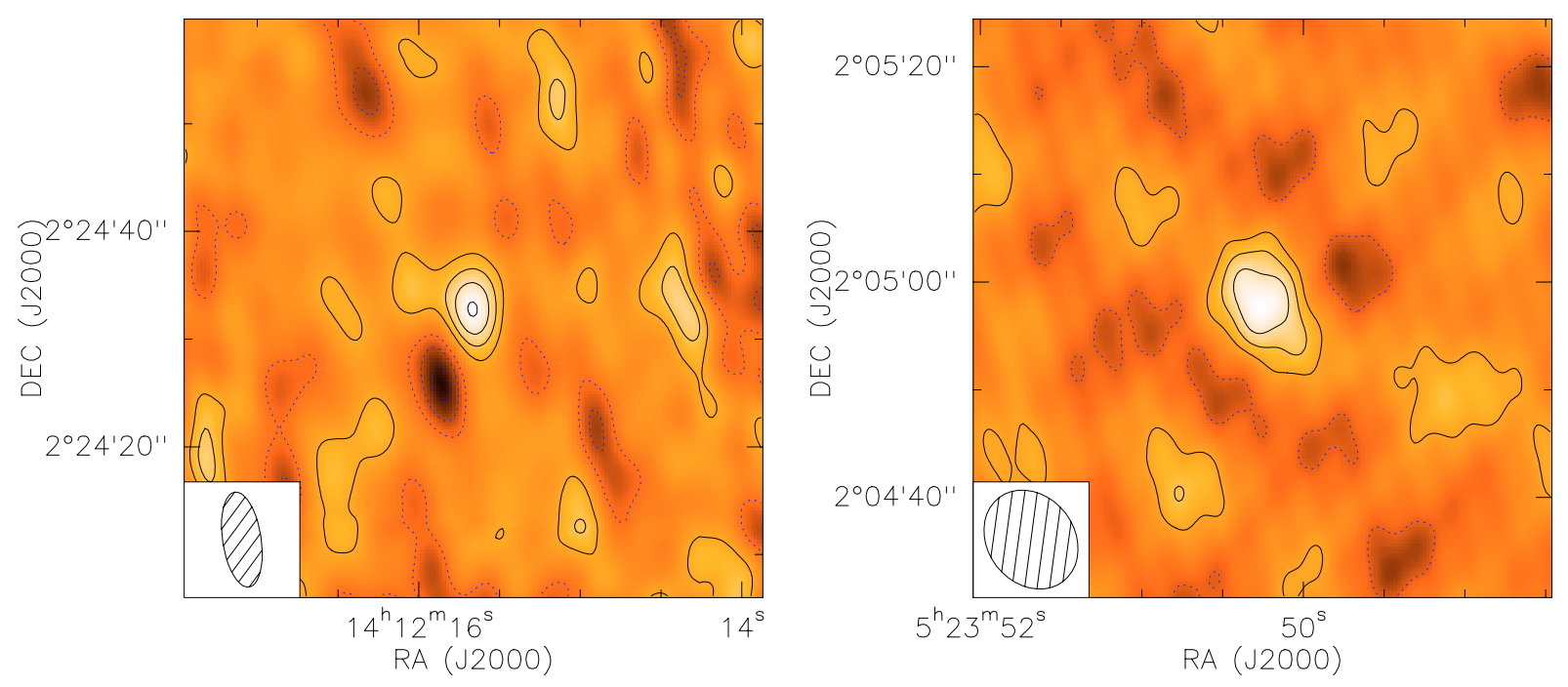

Fig. 1. HD 124224 (left panel) and HD 35298 (right panel) were detected with $\sim 4 \sigma$ in a beam of $8.9^{\prime \prime} \times 3.6^{\prime \prime}$ at PA $=9^{\circ}$ and $9.1^{\prime \prime} \times 8.1^{\prime \prime}$ at $\mathrm{PA}=37^{\circ}$, respectively (shown as dashed areas). Contours start at $1 \sigma$ and are in steps of $\sigma=0.43 \mathrm{mJy}$ for HD 35298 and $\sigma=0.31 \mathrm{mJy}$ for HD 124224.

field and EOIT in excess of $140 \mathrm{~h}$, no emission could be detected from HD 215441.

\section{Proposed mechanisms for the radio emission}

The photospheric magnetic field of MCP stars is mainly dipolar, even if higher multipoles can be important (Landstreet \& Mathys 2000). In the circumstellar regions, the dipolar component is dominant.

Drake et al. (1987) proposed that the radio emission from MCP stars is gyrosynchrotron emission from mildly relativistic electrons trapped in a belt surrounding the equator of the magnetic dipole. In the case of HD 37017 and HD 37479 the dimension of the magnetic cavity is $2 \leq R / R_{\star} \leq 10$. André et al. (1988) noted that the characteristics of the magnetosphere of MCP stars, as quantified by Havnes \& Goertz (1984), are not compatible with the emission model suggested by Drake and co-workers. André and co-workers suggested that the stellar wind, flowing along the field lines, draws these into current sheets, where electrons can be accelerated up to relativistic energies. Gyrosynchrotron emission is due to non-thermal electrons (whose energy distribution is $N(E) \propto E^{-\delta}$ ) located far from the star where the magnetic field is $10-100 \mathrm{G}$. Thus for a $10 \mathrm{kG}$ polar value of a magnetic dipole, the emitting region is located at $\sim 10 R_{\star}$. Adopting the previous scenario, Linsky et al. (1992) modelled the radio emission from HD 37479 assuming non-thermal electrons with a constant density and $\delta=2$. The disks with area equal to the emitting regions at 5 and $1.4 \mathrm{GHz}$ have radii equal to 3 and $8 R_{\star}$, respectively.

\section{Numerical simulations of the high frequency emitting regions}

We have computed the radio fluxes in the $1-90 \mathrm{GHz}$ range, on the basis of a detailed theory of gyrosynchrotron emission and absorption (Ramaty 1969). For our computations, we have considered homogeneous emitting regions with magnetic field $B=1,2,5$ and $7 \mathrm{kG}$ whose inclination angle with resepct to the line of sight is $\theta=20^{\circ}, 45^{\circ}$ and $80^{\circ}$; electron energy distribution with exponent $\delta=1,2,3$ and 4; thermal electron density $N_{\mathrm{Th}}=10^{8} \mathrm{~cm}^{-3}$; thickness of the emitting region $L$ equal to 0.01 and 0.001 solar radii.

Figure 2 shows the computed radio spectra normalised to the $22.5 \mathrm{GHz}$ value. For comparison, the spectrum of HD 124224, our best observed target, is also shown in this figure.

From the observed ratio between the radio fluxes at $87.7 \mathrm{GHz}$ and $22.5 \mathrm{GHz}$, we conclude that circumstellar regions, close to HD 124224 where the field assumes several $\mathrm{kG}$ values, cannot be responsible for gyrosynchrotron emission. Panels a and b of Fig. 2 show that the radio emitting region cannot have a magnetic field larger than $\sim 2 \mathrm{kG}$. From the observed radio spectra, we note that the $N_{\text {non-Th }} \times L$ cannot be much smaller than $\sim 10^{13} \mathrm{~cm}^{-2}$ and $\delta<3$ (Figs. 2a-c). Our results are not significantly dependent on the angle between the line of sight and the magnetic field (Fig. 2d).

\section{Radio spectrum}

Assuming an upper limit for the millimetre emission equal to $4 \sigma$, Fig. 3 shows the observed spectra for those stars with complete 1.4-87.7 GHz coverage (VLA observations are from Leone et al. 1996). HD 124224, HD 37017 and HD 37479, which are MCP stars with relatively weak magnetic fields (Table 1), have positive spectral index $\alpha\left(S_{v} \propto\right.$ $v^{\alpha}$ ) up to $22.5 \mathrm{GHz}$ beyond which there is a drop in the flux. HD 142301 and HD 215441, with much stronger magnetic fields, are characterised by negative spectral index up to $15 \mathrm{GHz}$ and were not detected at $22.5 \mathrm{GHz}$. The sharp decrement of the radio flux in a so small frequency interval could be indicative of a cut-off frequency.

Only two measurements of the $5 \mathrm{GHz}$ flux density are reported by Linsky et al. (1992) for HD 35298: $0.28 \pm 0.06$ and $<0.19 \mathrm{mJy}$. Unfortunately, only an average value for a set 

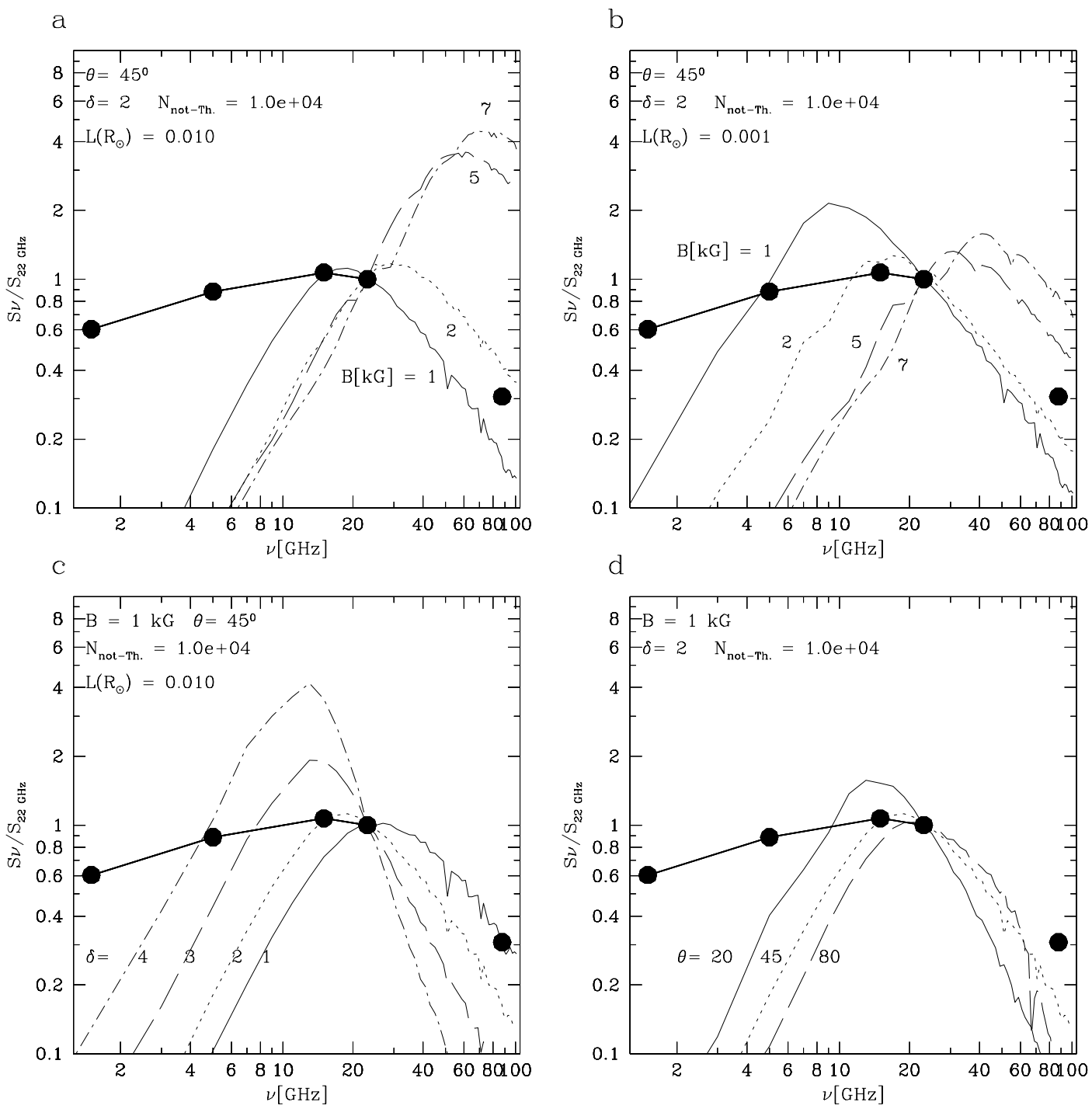

Fig. 2. Emerging flux distributions from a homogeneous source as computed for $B=1,2,5$ and $7 \mathrm{kG} ; \delta=1,2,3$ and $4 ; \theta=20,45$ and $80^{\circ}$; non-thermal electron density $N_{\text {non-Th }}=10^{4} \mathrm{~cm}^{-3}$; thickness of the emitting region equal to $L=0.01$ and 0.001 solar radii. Dots are flux densities observed towards HD 124224 in the cm range by Leone et al. (1996) plus the here measured $87.7 \mathrm{GHz}$ flux.

of five measurements of the effective field is available in the literature: $2.3 \pm 0.4 \mathrm{kG}$ (Borra 1981) and we therefore cannot estimate the polar value of the field for this MCP star. For this reason, the behaviour of this MCP star, whose radio spectrum seems to be the only one increasing from the $\mathrm{cm}$ $\left(S_{5 \mathrm{GHz}}=0.28 \mathrm{mJy}\right)$ up to the $\mathrm{mm}\left(S_{87.7 \mathrm{GHz}}=1.61 \mathrm{mJy}\right)$, cannot be related to its surface magnetic field strength.

In the framework of the Linsky et al. model, the gyrosynchrotron radiation at frequency $v$ is emitted from two opticallythick tori located symmetrically above the magnetic poles. Frequency and field strength are related by $B_{v} \propto v / s$ ( $s$ is the harmonic number) and the dipolar component of magnetic field (which dominates far from the star) decreases as $B \propto B_{\mathrm{p}} r^{-3}$, with $B_{\mathrm{p}}$ polar magnetic field and $r$ the distance from the star in stellar radii units. The emerging flux is given by

$S_{v} \propto \Omega_{v} I_{v}$

where $\Omega_{v}$ is the solid angle subtended by the emitting region and $I_{v}$ is the specific intensity. Pole-on projections of the emitting regions show up as circular shell with radius $r_{v} \propto$ $\left[B_{\mathrm{p}} / \nu\right]^{1 / 3}$ and thickness $\Delta r \propto r \Delta B / B$, hence

$S_{v} \propto r_{v}^{2} I_{v} \propto B_{\mathrm{p}}^{2 / 3} v^{-2 / 3} I_{v}$.

Since in the optically-thick approximation the specific intensity depends only on the frequency, the spectral index does not depend on the dipole strength. We conclude that the observed behaviour of the radio spectra is not consistent with the hypothesis of an optically thick region.

\section{Conclusions}

We have performed observations at $87.7 \mathrm{GHz}$ with the IRAM interferometer on a sample of eleven MCP stars that have been previously observed with the VLA in the cm-range. Only two of these stars, namely HD 35298 and HD 124224, yielded a detection $(\sim 4 \sigma)$ at mm-wavelengths.

Whenever possible, we have combined VLA and IRAM observations to cover the $1.4-87.7 \mathrm{GHz}$ range and found that 


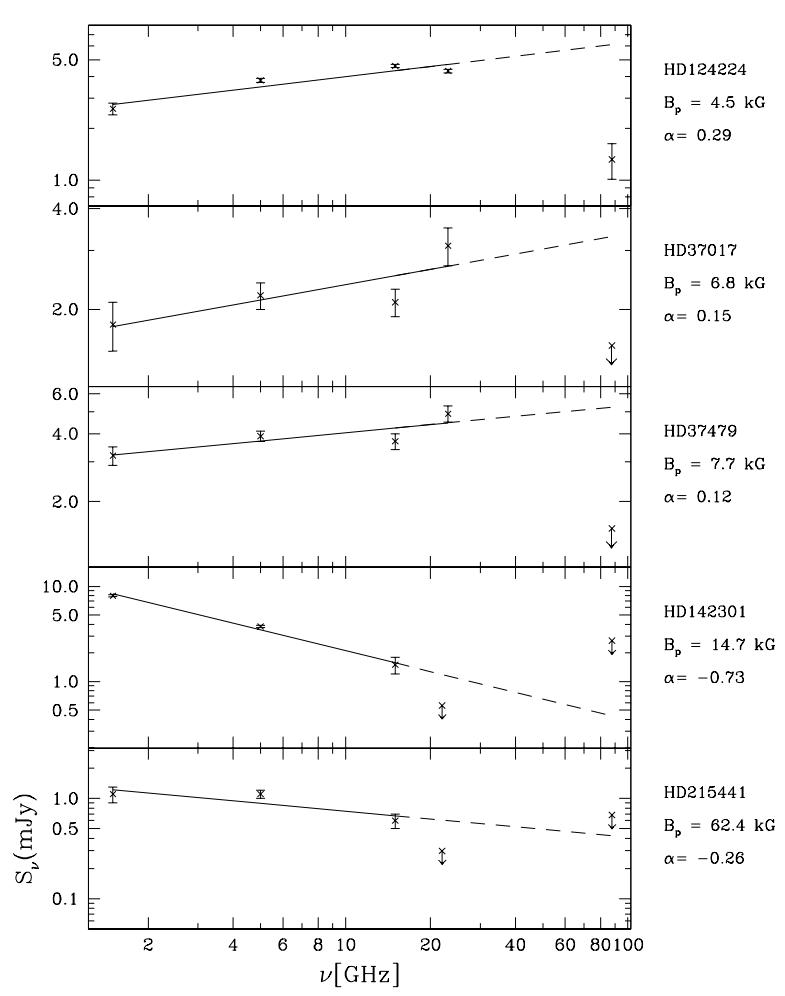

Fig. 3. Spectral energy distribution at $1.4,5,15,22.5$ and $87.7 \mathrm{GHz}$ for those stars with a complete VLA spectrum. The continuous line is a linear fit, with spectral index $\alpha$, of the centimetre emission in the log-log plane. The dashed line is its extrapolation toward the mm-range. Arrows represent upper limits for the observed flux densities and are defined as four time the rms listed in Table 1. Stars show an inversion of $\alpha$ when plotted for increasing value of the magnetic field from the top to the bottom.

the flux densities of the MCP stars HD 37017, HD 37479 and HD 124224, with relatively weak magnetic fields, increase with the frequency, up to a $v>22.5 \mathrm{GHz}$, and then decrease in the $\mathrm{mm}$ range. On the other hand, the radio emission from HD 142301 decreases steadily with frequency. Only HD 215441 shows an almost flat spectrum between 1.4 and $15 \mathrm{GHz}$ and no emission at the highest frequencies.

The radio spectra of HD 142301 and HD 215441 (with the strongest magnetic fields) exhibit a sharp drop in flux density between 15 and $22.5 \mathrm{GHz}$ which suggests there is a cut-off frequency in this range. On the other hand, the MCP stars with relatively weak magnetic fields show flux densities that certainly decrease from the $\mathrm{cm}$ to the $\mathrm{mm}$ range. However, it is not clear if the spectra have a smooth decrement, as it is for HD 215441 at about $5 \mathrm{GHz}$, or also a cut-off.

Further observations of HD 37017, HD 37479 and HD 124224 are necessary in the 22.5-87.7 GHz range to establish the presence of a possible cut-off frequency.
Also HD 35298 should be observed in the 5-87.7 GHz range to ascertain if its spectrum is really increasing from the $\mathrm{cm}$ to the $\mathrm{mm}$ range. A determination of its photospheric magnetic field is also necessary to verify the apparent anti-correlation between slope of the radio spectrum and photospheric magnetic field strength.

Numerical simulations based on the exact emission and absorption coefficients by Ramaty (1969) for the gyrosynchrotron emission show that the ratio between the 22.5 and $87.7 \mathrm{GHz}$ fluxes of HD 124224 is indicative of emitting regions where the magnetic field is less than a few $\mathrm{kG}$.

To explain the observed centimetre radio emission, it has been suggested that non-thermal electrons are created where the stellar wind draws the field lines into current sheets and that these electrons travel toward the star and emit gyrosynchrotron radiation. Because of the sharp decrease in the strength of a dipolar field with stellar distance, any frequency would be mainly emitted in a well localised torus above the magnetic poles. However, the observed slopes of the radio spectra and the absence of the millimetre emission are not compatible with this model. The sharp drop seen in the radio spectra of HD 142301 and HD 215441 beyond $15 \mathrm{GHz}$ is particularly difficult to explain.

Acknowledgements. We thank the IRAM staff for having carried out the observations and for help provided during the data reduction.

\section{References}

André, P., Montmerle, T., Feigelson, E. D., Stine, P. C., \& Klein, K.-L. 1998, ApJ, 335, 940

Borra, E. F. 1981, ApJ, 249, L39

Bohlender, D. A., Walker, G. A. H., \& Bolton, C. T. 1991, J. R. Astron. Soc. Can., 85, 202

Bohlender, D. A., Landstreet, J. D., \& Thompson, I. B. 1993, A\&A, 269,355

Cassinelli, J. P. 1984, Evidence for non-radiative activity in hot stars, in The Origin of Nonradiative Heating/Momentum in Hot Stars, ed. A. B. Underhill, \& A. G. Michalitsianos, NASA CP-2358, 2

Drake, S. A., Abbot, D. C., Bastian, T. S., et al. 1987, ApJ, 322, 902

ESA 1997, The Hipparcos Catalogue, ESA SP-1200

Havnes, O., \& Goertz, C. K. 1984, A\&A, 138, 421

Guilloteau, S., Delannoy, J., Downes, D., et al. 1992, A\&A, 262, 624

Landstreet, J. D., Borra, E. F., \& Fontaine, G. 1979, MNRAS, 188, 609

Landstreet, J. D., \& Mathys, G. 2000, A\&A, 359, 213

Leone, F. 1991, A\&A, 252, 198

Leone, F., Umana, G., \& Trigilio, C. 1996, A\&A, 310, 271

Linsky, J. L., Drake, S. A., \& Bastian, T. S. 1992, ApJ, 393, 341

Ramaty, R. 1969, ApJ, 158, 753

Trigilio, C., Leto, P., Leone, F., Umana, G., \& Buemi, C. 2000, A\&A, 362, 281 